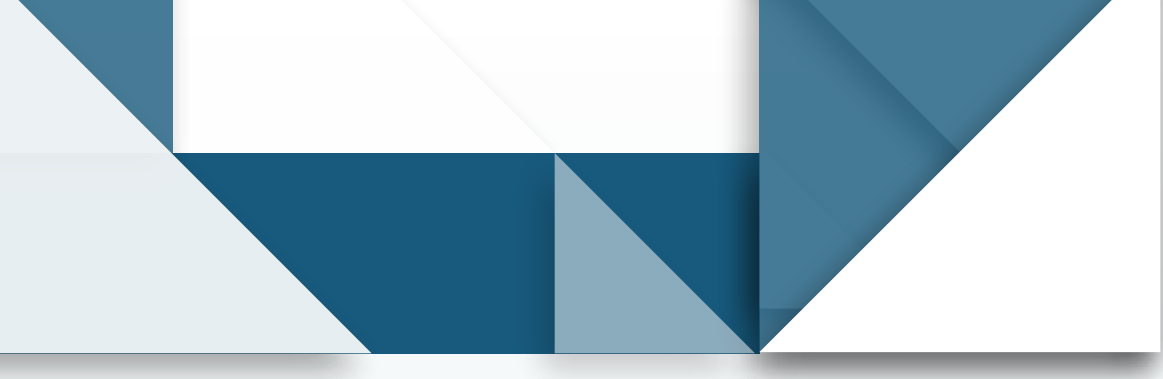

\title{
La historia oral en las \\ 17 memorias de prácticas \\ culturales para pensar un (por)venir de la educación (matemática) indígena
}

- Oral History in the Memories of Cultural Practices to Think about the Future of Math Education among Indigenous People Abelardo Tascón ** Diana Jaramillo**

- A história oral nas memórias de práticas culturais para pensar um (por)vir da educação (matemática) indígena

\section{Resumen}

Este artículo presenta el proceso de investigación titulado "La movilización de objetos culturales desde las memorias de la práctica de construcción de la vivienda tradicional Embera Chamí: posibilidades para pensar el (por)venir de la educación (matemática) indígena" y pretende visibilizar las tensiones y desafíos que implica el hacer investigación, y ser investigador, en el campo de la Etnomatemática. De esta manera se presenta tres tensiones, la primera, respecto a los vínculos relacionales entre las investigadoras y la comunidad indígena. La segunda, referida a la necesidad de considerar la lengua y los lenguajes producidos por las comunidades para ampliar los horizontes teóricos, metodológicos y prácticos del proceso de investigación. La tercera, en la apertura personal en aprender otras formas de ser y de estar en el mundo. La investigación se desarrolla desde el método de historia oral, el cual, valora y comprende la experiencia desde las voces de quienes han sido silenciados y/u oprimidos, tiene como elemento base el análisis deconstructivo. Como resultado, se consigue que los Jaibanás, (personas dedicadas a proteger el territorio y la salud de los miembros de la comunidad), participen, proponiendo alternativas para que parte de la sabiduría Embera Chamí fuese explicitada, discutida y problematizada en la escuela.

Palabras clave

Educación matemática; educación indígena; etnomatemática; comunidad Embera Chamí; análisis deconstructivo

\section{Abstract}

This paper presents the research process titled "The mobilization of cultural objects from the memories of the practice of construction of traditional Embera Chamí houses: possibilities to think about the future of math education among
Magíster en Educación, Línea de Formación en Educación Matemática, Profesora ocasional de la Universidad de Antioquia, Colombia, Medellín. Correo electrónico: carolina.higuita@udea. edu.co

orcid.org/0000-0002-6670-767X

** Licenciado en Pedagogía de la Madre Tierra, profesor indígena de la Comunidad Embera Chami, Escuela Rural Indigenista La María, Colombia, Valparaíso. Correo electrónico: atabelardo1@ gmail.com

orcid.org/0000-0003-3339-1776

*** Doctora en Educación, área Educación Matemática, profesora vinculada de la Universidad de Antioquia, Colombia, Medellín. Correo electrónico: diana.jaramillo@udea.edu.co orcid.org/0000-0003-1192-5642 
indigenous people", and it aims to show the tensions and challenges involved in researching and being a researcher in the field of ethnomathematics. Thus, the article presents three tensions: The first one has to do with the relational links between the researchers and the indigenous community. The second one is related to the need to consider language and the languages produced by the communities to broaden the theoretical, methodological and practical horizons of the research process. The third one has to do with the personal openness to learn other ways of being and existing in the world. The investigation follows the oral history method, which values and understands the experience from the voices of those who have been silenced and/or oppressed, and it is based on deconstructive analysis. As a result, we get the Jaibanás (those in charge of protecting the territory and health of the members of the community) to participate, proposing alternatives for some of the Embera Chamí wisdom to be made explicit, discussed and problematized at school.

\section{Keywords}

Math education; indigenous education; ethnomathematics; Embera Chamí community; deconstructive analysis

\section{Resumo}

Este artigo apresenta o processo de pesquisa intitulado "A mobilização de objetos culturais desde as memórias da prática de construção da vivenda tradicional Embera Chamí: possibilidades para pensar o (por)vir da educação (matemática) indígena" e pretende visibilizar as tensões e desafios que implica realizar pesquisa, e ser pesquisador, no campo da Etnomatemática. Dessa forma, apresentam-se três tensões, a primeira, com relação aos vínculos relacionais entre as pesquisadoras e a comunidade indígena. A segunda, com relação á necessidade de considerar a língua e as linguagens produzidas pelas comunidades para ampliar os horizontes teóricos, metodológicos e práticos dos processo de pesquisa. A terceira, na apertura pessoal em aprender outras formas de ser e estar no mundo. A pesquisa é desenvolvida desde o método de história oral, que valora e compreende a experiência desde as vozes daqueles que foram silenciados ou oprimidos, tem como elemento base a análise desconstrutiva. Como resultado, os Jaibanás (pessoas dedicadas a proteger o território e a saúde dos membros da comunidade), participem propondo alternativas para que parte da sabedoria Embera Chami seja explicitada, discutida e problematizada na escola.

Palavras-chave

Educação matemática; educação indígena; etnomatemática; comunidade Embera Chami; análise desconstrutiva 


\section{Una introducción}

La investigación intitulada "La movilización de objetos culturales desde las memorias de la práctica de construcción de la vivienda tradicional Embera Chamí: posibilidades para pensar el (por)venir de la educación (matemática) indígena" (Higuita, 2014) se situó en una perspectiva histórico-cultural de la educación matemática; más específicamente en la etnomatemática y en la educación indígena. La pregunta orientadora de este trabajo fue: ¿ Cómo se movilizan objetos culturales desde las memorias de la práctica de la construcción de la vivienda tradicional embera chamí para pensar el (por)venir de la educación (matemática) indígena? Así, los objetivos de estudio fueron: en primer lugar, analizar la movilización de objetos culturales desde las memorias de la práctica de la construcción de la vivienda tradicional embera chamí; y, en segundo lugar, problematizar esa movilización de objetos culturales para pensar el (por)venir de la educación (matemática) indígena.

En esta investigación, la práctica cultural la comprendimos, de acuerdo con Miguel (2010):

Conjunto articulado e intencional de acciones individuales o colectivas que derivan sus significaciones del juego discursivo constituido en la (y constitutivo de la) actividad que define la existencia social de esa práctica y de la propia comunidad que la realiza. Tales prácticas son siempre condicionadas por instituciones sociales, esto es, por conjuntos dinámicos de normas de cualquier naturaleza que organiza y condiciona (restringiendo, posibilitando o delimitando) las relaciones sociales al interior de esa comunidad, así como todos los demás tipos de relaciones simbólicas -en ellas incluidas las relaciones de poderque los sujetos establecen entre sí, con la naturaleza y con el conjunto de artefactos mediadores de esas relaciones. (p. 14).
Para alcanzar los objetivos propuestos construimos, de manera colectiva, el camino metodológico, el cual implicó el diálogo, fundamentalmente, con participantes de la comunidad embera chamí, y, con autores como: D'Ambrosio (1999; 2008; 2011$)$, Miguel $(2008 ; 2010)$, Thompson (2011), Walsh (2005; 2007a; 2007b), entre otros.

Los registros producidos de manera conjunta entre los participantes de la investigación -todos indígenas embera chamí- y las investigadoras se originaron desde: el diario de investigación; fichas temáticas; encuentros con el Cabildo'; encuentros con el Círculo de Jaibanás ${ }^{2}$ (figuras 1 y 2); encuentros con jóvenes; entre-vistas con los maestros indígenas. La entre-vista fue comprendida en la investigación, en coherencia con lo propuesto por Kvale (201 1), como "una visión-entre, un intercambio de visiones entre dos personas que conversan sobre un tema de interés común" (p. 27).

Según el artículo 330 de la Constitución Política de Colombia de 1991, los cabildos son consejos "conformados y reglamentados según los usos y las costumbres de sus comunidades y ejercerán las siguientes funciones: (1) Velar por la aplicación de las normas legales sobre usos del suelo y poblamiento de sus territorios. (2) Diseñar las políticas y los planes y programas de desarrollo económico y social dentro de su territorio, en armonía con el Plan Nacional de Desarrollo. (3) Promover las inversiones públicas en sus territorios y velar por su debida ejecución. (4) Percibir y distribuir sus recursos. (5) Velar por la preservación de los recursos naturales. (6) Coordinar los programas y proyectos promovidos por las diferentes comunidades en su territorio. (7) Colaborar con el mantenimiento del orden público dentro de su territorio de acuerdo con las instrucciones y disposiciones del Gobierno Nacional. (8) Representar a los territorios ante el Gobierno Nacional y las demás entidades a las cuales se integren; y (9) Las que les señale la Constitución y la ley.

Parágrafo: la explotación de los recursos naturales en los territorios indígenas se hará sin desmedro de la integridad cultural, social y económica de las comunidades indígenas. En las decisiones que se adopten respecto de dicha explotación, el Gobierno propiciará la participación de los representantes de las respectivas comunidades".

2 Los jaibanás son los médicos tradicionales de la comunidad; son quienes curan los dolores del cuerpo y del espíritu de los indígenas embera chamí; ellos cuidan y protegen el territorio donde habita la comunidad. 


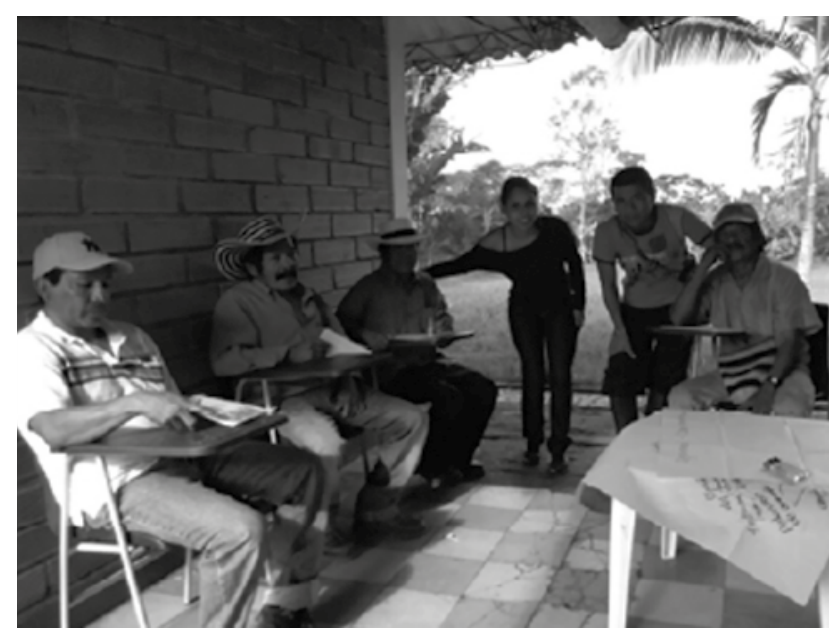

Figura 1. Primer Círculo de Jaibanás, 10 de mayo de 2013

Fuente: Juan Carlos Jamioy Juangibioy.

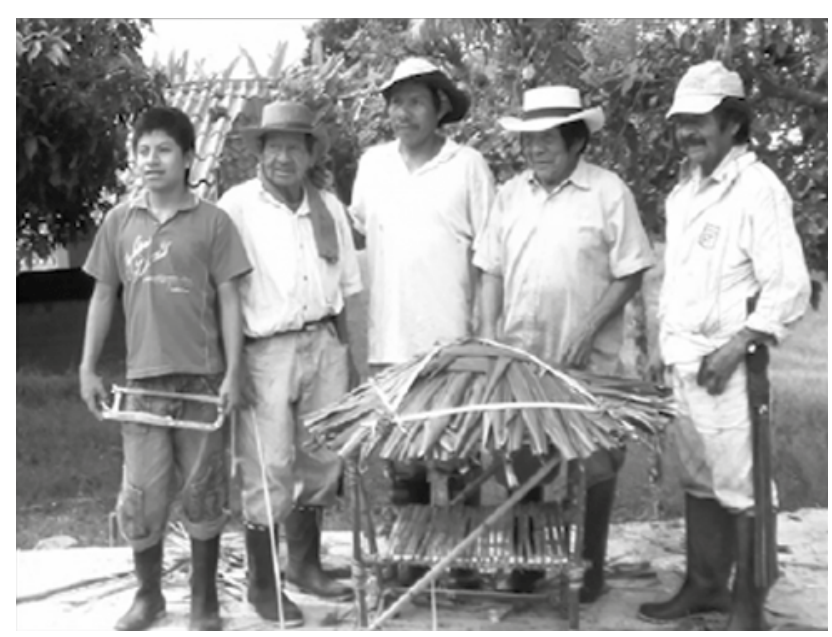

Figura 2. Tercer Círculo de Jaibanás, 19 de julio de 2013

Fuente: Juan Carlos Jamioy Juangibioy.

En el análisis deconstructivo de estos registros hicimos visible, en primer lugar, un posicionamiento cultural, político y simbólico de la comunidad indígena embera chamí desde la movilización de objetos culturales tales como medida, número y forma ${ }^{3}$; posibilitando, así, reflexiones para pensar un (por)venir de la educación (matemática) indígena. Y, en segundo lugar, la comprensión de que a medida que dicha movilización de objetos culturales se realizaba, se dio un proceso de identificación de la comunidad, no solo con un otro indígena, sino también con un otro no indígena. De esta manera, resaltamos la importancia de que al ser los objetos culturales movilizados por los sujetos, estos mismos objetos culturales movilizaron al sujeto. 
En este artículo pretendemos visibilizar, desde nuestra experiencia investigativa, las tensiones y desafíos que implica el hacer (y el ser) investigación en el campo de la etnomatemática. Tensiones y desafíos evidenciados desde las posibilidades que brinda la historia oral -método utilizado en este trabajo-. Para ello, compartimos las reflexiones que desde los aspectos metodológicos de la investigación fueron emergiendo. Aspectos que estuvieron en dialéctica con otros de orden epistemológico, ontológico y gnoseológico.

\section{Antecedentes y marco teórico}

\section{Un inicio del camino recorrido}

Iniciar una investigación en etnomatemática siempre está acompañado de miedos, alegrías y, sobre todo, incertidumbres, entendidas estas últimas como recorrer caminos desconocidos, en donde lo que planeamos y soñamos puede o no darse. Ello, porque esos caminos no dependen, apenas, del investigador; son caminos que también dependen de las historias, de los intereses y de los compromisos de las personas que vienen tejiendo ese camino. Al mismo tiempo, la investigación depende de la dinámica social, económica, política y cultural del país donde ella está inmersa. Incertidumbres y dependencias que fuimos conociendo en el camino de esta investigación.

En este aparte queremos mostrar los ires y venires de la investigación. En lengua embera chamí, el símbolo que expresa la palabra camino es $\theta$, el cual puede interpretarse como un proceso cíclico, a partir de algo que se tiene como origen. Ese proceso cíclico implica ir y venir; implica, a veces, volver a donde se estaba inicialmente, incluso alejarse otra vez, pero teniendo siempre presente qué es lo que motiva el recorrer los caminos por venir.
De los significados de la palabra camino, expresados en el diccionario de la Real Academia Española (RAE, 2013), dos se aproximaron a aquello que nosotros vivimos y compartimos. Nos referimos a: "vía que se construye para transitar" y "dirección que ha de seguirse para llegar a algún lugar". Decimos que se aproximaron, pues a veces no era fácil construir esa vía, seguir esa dirección. A veces creíamos construir un camino, pero, alguien o algo, como lo expresa Larrosa (2009), nos ponía a dudar, nos cuestionaba. Ese alguien o algo, de acuerdo con ese autor, se refiere a lo "que es exterior a mí, extranjero a mí, extraño a mí, que está fuera de mí mismo, que no pertenece a mi lugar, que no está en el lugar que yo le doy, que está fuera de lugar." (p. 15).

En este sentido, el camino en esta investigación no fue construido por una sola persona, sino a partir de las creencias, de los intereses, de la sabiduría de las personas que se unieron a esta idea de pensar una educación otra para la comunidad embera chamí. Tratamos de dialogar -desde las diferentes formas de hacer y (de ser) de los participantes de este estudio- con el objetivo común de buscar alternativas posibles para el desarrollo del campo educativo indígena.

Así, la metáfora del camino ayudó a describir, como lo sugiere Smith (2012), los encuentros y los desencuentros que pudieron ocurrir en la investigación con esta comunidad indígena porque, como sucedió en este caso, se dio un diálogo y un caminar entre indígenas Embera chamí y mujeres que no somos indígenas (kapuria wera, en lengua embera) ${ }^{4}$.

Esas mujeres no indígenas éramos la investigadora principal, Carolina Higuita Ramírez, y la orientadora de la investigación, Diana Jaramillo. 


\section{Tensiones en la investigación}

\section{Relaciones de las investigadoras con la comunidad}

Comprendemos con Smith (2012) que "la investigación es un espacio de confrontación no sólo en el nivel de la epistemología o de la metodología sino también, en su sentido más amplio, como actividad académica profundamente conectada con el poder" (p. 194). Esta comprensión fue importante en este estudio, pues en él partimos del reconocimiento y de la valoración del lugar que ocupaba cada una de las personas participantes. Ese reconocimiento no fue entendido apenas como una cuestión de protocolo investigativo, asociado a aspectos éticos de la investigación; sino que fue asumido principalmente como un elemento clave referido a aquello que debe, puede o quiere decirse mientras se participa de una investigación. Esto es, ese reconocimiento se entendió como un acto de poder.

A las investigadoras, que representábamos una institución -en este caso la Universidad de Antioquia, desde nuestra participación en la Maestría en Educación, Línea de Formación en Educación Matemática y, al mismo tiempo, como profesoras de la Licenciatura en Pedagogía de la Madre Tierra (LPMT) ${ }^{5}$ - se nos posibilitó una aceptación en la comunidad indígena embera chamí para proponer, discutir y desarrollar de manera conjunta, con ellos, la investigación. A lo anterior puede unirse el hecho que ya habíamos participado en investigaciones anteriores con la comunidad, sobre las cuales se presentaron algunos resultados en términos de reflexiones que posibilitaron discusiones en el ámbito educativo (Berrío 2009; Higuita, 2011 ).

Este reconocimiento que como investigadoras teníamos dentro de la comunidad, no desconocía que nosotras éramos mujeres de otra cultura, con otras historias, con otras creencias, con otras formas diferenciadas de pensar y de concebir la educación y el mundo. Sin embargo, la comunidad sabía también que éramos mujeres sensibles a las realidades de los pueblos indígenas. De esta manera, afirmamos que ya existía -en nosotras- una familiaridad con los maestros y los líderes de la comunidad; así mismo, afirmamos que existía un conocimiento nuestro de las dinámicas, problemáticas y luchas que la comunidad venía urdiendo.

5 La Licenciatura en Pedagogía de la Madre Tierra es un programa de formación que forma parte de la Facultad de Educación de la Universidad de Antioquia. Su legalización se dio partir de la Resolución Académica 1752 del 18 de agosto de 2005, expedida por esta misma entidad; y obtuvo, desde el Ministerio de Educación Nacional de Colombia (MEN), su registro calificado No. 513 el 01 de febrero de 2011 . El programa tiene por objetivo cuestionar y atender las problemáticas que en el ámbito educativo se presentan en las diferentes comunidades indígenas de Antioquia desde la formación de maestros indígenas. Este programa de formación, en su primera cohorte, se propuso para los cinco pueblos indígenas de Antioquia; son ellos: senú, dule, embera chamí, embera eyábida (o katío) y embera dóbida. El ciclo básico de la Licenciatura que comprende los tres primeros años toma como ejes temáticos los siguientes: "Reencuentros con nuestros saberes y nuestras realidades"; "Diálogo con los otros", y "El futuro que soñamos". Los dos últimos años forman parte del ciclo de profundización, propuesto desde tres énfasis: "Lenguajes e interculturalidad"; "Ordenamiento y autonomía territorial"; y, "Salud comunitaria intercultural". La razón de estos énfasis obedece fundamentalmente a las necesidades que van teniendo las comunidades indígenas en estas áreas. 


\section{El lenguaje, un aspecto constituyente en la investigación}

No solamente el saber a quién se hablaba y con quién se dialogaba era fundamental para la investigación; también lo era cómo se hablaba y cuál sería la lengua a ser utilizada. En este sentido, como investigadoras conocíamos nuestras fortalezas y nuestras limitaciones. Saber que no dominábamos la lengua embera chamí era una limitación, toda vez que era la lengua en la que los participantes podían expresarse de manera fluida y espontánea (aunque vale decir que varios de los indígenas embera chamí también son hablantes de la lengua castellana).

La identificación de este aspecto era importante. Sin embargo, no dominar la lengua tampoco se convirtió en un impedimento para la realización de la investigación. Dentro de la comunidad existían intérpretes (algunos maestros y algunos líderes) de reconocida confianza por las personas con las que conversamos. Intérpretes que nos posibilitaron tener una comprensión de lo dicho, tanto desde la lengua misma como desde la traducción ${ }^{6}$ al castellano.

Lo anterior era importante porque, tanto a nivel metodológico como epistemológico, teníamos que tener cuidado, como lo dice Kvale (2011), en "seleccionar un intérprete que sea aceptable culturalmente y además domine el idioma. El papel del intérprete es ayudar y no asumir el papel del entrevistador o del entrevistado" (p. 96). Dentro de la comunidad, los intérpretes cumplieron un papel importante, especialmente cuando se dieron las conversaciones con los jaibanás y algunos ancianos.

Este aspecto de la lengua indígena embera chamí no podía ser considerado como uno más en este estudio. Se tornó clave, tanto en el desarrollo de la investigación como en las

6 Traducir en el sentido sugerido por Larrosa (1998): traducir es interpretar. reflexiones compartidas; ello, por las posibilidades ofrecidas al hablar en lengua nativa para una comunicación fluida y una generación de ideas y de saberes. En ese sentido, fue evidente, para nosotras, y como lo expresa Chilisa (2012), que el uso de la lengua nativa en la investigación contribuye en "la promoción de nuevos conocimientos, nuevos conceptos, nuevas teorías, nuevas normas, métodos y técnicas de investigación" (p. 57). Fue desde la lengua embera chamí, desde la palabra indígena, donde se encontraron formas otras de enunciar, de caminar, de pensar.

\section{La posibilidad de aprender formas otras de ser y formas otras de estar en el mundo}

La propuesta de desarrollar un proyecto desde, para y con la comunidad embera chamí, a partir del estudio de la práctica de la construcción del purradé ${ }^{8}$ para pensar la educación (matemática) indígena generó, entre los líderes de esta comunidad, una sensación de que alguien había venido a recordar un compromiso propio que la comunidad estaba aplazando por múltiples razones. Esta sensación motivó a los líderes para apoyar el proyecto y sirvió, además, para reconocer que ya se venían tejiendo algunas reflexiones en torno a esta práctica al interior de la comunidad.

Así, el proyecto se constituyó en la continuación de lo que se venía realizando o soñando, y en un cambiar la mirada respecto a lo realizado. En este sentido, Julio Vélez, gobernador del Cabildo, expresó lo siguiente:

7 La expresión estar en el mundo es tomada de Freire (2004). Con ella, el autor nos indica que, en nuestro papel como formadores e investigadores, tenemos que tomar decisiones, asumir posiciones y hacer rupturas cuando sea necesario; rupturas de orden cultural, social y político que nos posibiliten diversas formas de intervenir en el mundo.

8 Purradé es el nombre dado en lengua embera chamí a la vivienda tradicional. Puede verse una maqueta de él en la figura 2. 
Vamos a ver: yo entiendo que lo que se busca con esto es cómo recuperar todas las costumbres nuestras, ¿̇cierto? No solo porque la profesora Carolina esté acá, sino que, igualmente, al interior de la comunidad tenemos que pensar nosotros en lo que son las propiedades nuestras y nuestras costumbres. No sé por qué, por qué motivo, hasta el son de hoy, no hemos vuelto a recuperar lo que se cayó. Yo, inclusive ahora, cuando vino la profe Carolina, yo le decía: "Mire que el tambo9 estaba allí, en aquellos lados, y eso se cayó y ya; hasta ahí llegó". Pero, igualmente, la comunidad y el Cabildo ya habían pensado en eso. Eso se cayó; y no se cayó solo, pues nosotros lo íbamos a reformar, porque el techo estaba muy malo, había una madera que había que cambiar; entonces dijimos: "Tumbemos esto y hay que volver a levantarlo, hay que volver a recuperarlo nuevamente" y, hasta el son de hoy, no se ha vuelto a recuperar. Yo creo que, con la venida suya, con el proyecto que usted piensa, yo creo que, desde nosotros, más que desde usted Carolina, tenemos que ver en este proyecto el cómo volver a recuperar lo nuestro, lo que se nos está quedando, lo que se nos está perdiendo. (Primer encuentro con el Cabildo, Julio Vélez Tascón, 7 de abril de 2013).

La voz del gobernador del Cabildo mostró el apoyo a la propuesta en términos de una posibilidad de diálogo entre personas de la comunidad y externas a ella, para apuntar caminos sobre los cuales, todavía, había mucho que recorrer. Pero al mismo tiempo, esa voz mostró cómo esta investigación debía considerar y enfrentar situaciones que la comunidad venía percibiendo y viviendo desde tiempo atrás.

Lo que debía considerarse, en ese momento, era que ya se tenían algunos adelantos en cuanto a la reflexión de la práctica; esto es, era necesario conversar con esos otros miembros de la comunidad que ya se habían interesado por el tema desde su participación en la Licenciatura en Pedagogía de la Madre Tierra.

Debíamos también enfrentarnos a cómo se iba a realizar la investigación. Sobre este último aspecto, Ángel Miro Tascón, líder de la comunidad, expresó:

Yo no sé si usted Carolina ha construido alguna metodología de trabajo para que ese día [refiriéndose a un próximo encuentro con el Cabildo] lo pudiéramos socializar y mirar si se puede hacer ajustes a esa metodología o se puede dejarlo tal cual como nos la presenta. Eso sería como el segundo paso, construir la metodología y el plan de trabajo: ¿̇cuándo?, ¿̇dónde?, ¿̇cuándo empieza eso?, ¿̇qué actividades realizar?, ¿̇quién va a ser el encargado de todo esto?, ¿̇el Cabildo, usted, los profesores, los estudiantes de Madre Tierra, los líderes, los niños? Bueno, yo creo que eso es como el segundo paso a seguir: la metodología de las actividades. De hecho, le recomiendo Carolina que, para organizar esa idea de la reunión, usted nos puede mostrar alguna iniciativa de la construcción de esa metodología; así, la miramos, ya sea para hacer ajustes o para dejarla tal cual. Me imagino que sí deben haber algunas inquietudes para construir juntos una metodología de acuerdo a usos y costumbres de la comunidad. (Primer encuentro con el Cabildo, Ángel Miro Tascón, 7 de abril de 2013). 
Consideramos que el interés por cómo iba a ser desarrollada la investigación era consecuencia y causa del posicionamiento cultural y político que las comunidades indígenas de Antioquia vienen logrando en los últimos años para proteger, respetar, valorar y legitimar su propia sabiduría; de ahí el interés manifestado por el líder de sentirse y formar parte de la construcción de la metodología del estudio. Entendemos que para ese líder, eso se constituía en un hecho político, intentando dar un paso diferente para transformar una historia ya vivida. Una historia que era comprendida como lo expresa Smith (2012):

La historia de la investigación desde diversas perspectivas indígenas está tan profundamente incrustada en los procesos colonizadores que siempre la han visto solo como una herramienta que sirve exclusivamente a la colonización y no como una herramienta potencial para la autodeterminación y el desarrollo. Para los pueblos indígenas, el significado de la investigación no puede separarse, según nuestra historia como nativos, de la mirada de la ciencia occidental y del colonialismo. (p. 193).

No queríamos continuar esa historia. Se trataba ahora de recorrer otros caminos que, por lo menos, nos alejaran (a la comunidad y a nosotras investigadoras) de visiones esencialistas, absolutistas, colonizadoras y excluyentes de la investigación, en donde las comunidades y, en particular, los indígenas eran considerados meros informantes pasivos. Además, sabíamos que no sería fácil este recorrido, porque por años esas visiones de la investigación han primado, incluso dentro de la comunidad misma. Así, juntos, tuvimos la convicción de emprender otro trasegar metodológico, pues, en este caso, el poder de la palabra la tenían ellos, los miembros de la comunidad.
Necesitábamos aprender las formas en que, a su vez, los miembros de la comunidad aprendían en el contexto de las prácticas. Necesitábamos también aprender las formas en que ellos hacían investigación dentro de la comunidad y cómo habían sido educados. Sabíamos, como diría Rigney (citado por Smith, 2012), que teníamos que privilegiar "el conocimiento, las voces y las experiencias de los indignas, así como el análisis de sus condiciones sociales, materiales y espirituales" (p. 194). Tal vez, así, la investigación podía no solamente ser movilizada en la comunidad sino que, al mismo tiempo, posibilitar movilizaciones entre las personas que participábamos.

De esas otras formas de hacer (y ser) en investigación, aprendimos que existen cuatro principios clave para la comunidad embera chamí: observar; escuchar; recorrer y volver a recorrer.

\section{Metodología}

\section{La historial oral como una posibilidad para aproximarnos a la práctica de la construcción del purradé}

En el diálogo constante con la comunidad para construir la metodología, y particularmente con los maestros (participantes de la investigación), encontramos que su apuesta para estudiar la práctica y los objetos culturales allí movilizados apuntaba, necesariamente, al diálogo con los jaibanás y con las personas de mayor edad en la comunidad. En una conversación con el maestro, él nos dijo:

Sé que en la memoria de ellos [refiriéndose a los Jaibanás] está guardada toda esa información que de pronto uno no llegó a sacar de ellos o que ellos nunca dieron. Sé que con ellos, tanto con Rafael 
y con Miguel, podemos obtener unas buenas conversaciones, muy interesantes conversaciones. Incluso, yo sé que para usted, Carolina, y para mí va ser muy bueno. (Conversación, Abelardo Tascón, 3 de octubre de 2012).

Ese llamado insistente para hablar con los jaibanás tenía que ser considerado en la investigación. Se trataba de conversar, de tejer unas relaciones de confianza, a partir de las cuales las historias sobre la práctica de la construcción del purradé fueran emergiendo y, con ellas, toda la sabiduría presente. No era solo la voz del maestro indígena Abelardo; otras voces también expusieron la necesidad de hablar con los jaibanás. Por ejemplo, Jaime Alberto Tascón, líder de la comunidad, manifestó:

Con toda esa gente [refiriéndose a los jaibanás] usted puede sentarse, porque ellos tienen más conocimientos que nosotros, porque ellos sí lo vivieron [refiriéndose al hecho de vivir en el purradé]. Los jaibanás, por ejemplo, los médicos, el mismo don Ángel, todos esos jaibanás tienen mucha historia. (Segundo encuentro con el Cabildo, Jaime Alberto Tascón, 15 de abril de 2013).

Dentro de la comunidad había un interés particular en los jaibanás. Ellos tenían un papel clave, no solamente para proteger la vida y el territorio, sino, además, para recordar y para contar historias de la comunidad. Refiriéndose a la sabiduría de los mayores, Bosi (1994) expresa: "Habría, por tanto, para el viejo una especie singular de obligación social, que no pesa sobre los hombres de otras edades: la obligación de recordar, de recordar bien" (p. 63).

Esta función, la de recordar, asignada a los jaibanás, implicaba necesariamente narrar, contar historias. Históricamente, los pueblos indígenas desde la palabra han transmitido, de generación en generación, sus enseñanzas, sus tradiciones, sus prácticas. Hoy son ellos, las personas adultas, los jaibanás, quienes poseen esa sabiduría, quienes la pueden recrear. Sabiduría que se mantiene a pesar de que sus voces han sido, por años, silenciadas, excluidas por otras culturas o, en ocasiones, hasta por la propia cultura de la que forman parte.

Así, la investigación fue reconociendo como unidad de análisis las voces de los sabios, expresadas desde diversas escrituras (dibujos, símbolos, fotografías). Fueron precisamente esas voces las que nos posibilitaron entender y comprender la práctica, es decir, su sabiduría. Además nos posibilitaron encontrar caminos -metodológicos y epistemológicos- para recorrer o para volver a recorrer.

En este sentido, apuntando siempre a que eran los jaibanás quienes tenían la voz, a quienes teníamos que escuchar, consideramos que un método coherente con las necesidades y formas propias de actuar de la comunidad podría ser el método denominado de historia oral.

Esa consideración, porque la historia oral, desde Joutard (1999), Bosi (2003) y Sebe (2009): 
- Reconoce que desde la voz del otro, de los otros -generalmente aquellos que han estado silenciados- se proponen alternativas para comprender las realidades desde otras lógicas, desde otros órdenes diferentes a los establecidos.

- La historia es construida y reconstruida en tiempos y espacios locales, por tanto las culturas son móviles.

- La historia oral siempre parte de una preocupación del presente, esto es, desde el pasado. En ella el tiempo no puede ser comprendido como lineal. En las comunidades indígenas, por ejemplo, el presente es el pasado y el futuro es lo que está detrás. Así, para las comunidades, el tiempo es cíclico.

- La fuente oral, esto es, la palabra, al decir de Bosi (2003), "más que afirmar, camina en curvas y desvíos obligando a una interpretación sutil y rigurosa" (p. 20).

- Lo dicho y lo hecho deben ser explicados en el contexto en que fueron mencionados, realizados.

- Lo oral y lo escrito no se consideran como opuestos, sino como complementos en la comprensión de la realidad.

- Las memorias, esto es, las narrativas inventadas y reinventadas, son producto de la realidad actual, están impregnadas de las voces de sus antepasados, de sus ancestros; ellas constituyen sus identidades, pues como dice Joutard (1999), la historia oral "también pone de relieve el testimonio indirecto, no el de las personas que han vivido lo que cuentan sino el que transmite lo que les han dicho otros, es decir la tradición oral" (p. 210).
Este último aspecto que caracteriza la historia oral se convirtió para nosotros en un elemento fundamental. El desarrollo mismo de la investigación hacía visible la relación entre memorias, identidades y comunidad. Un aspecto que mereció nuestra especial atención.

La búsqueda de identidades a partir de las memorias pasa, necesariamente, por un análisis de lo actual, de lo que se está viviendo. La escogencia de los recuerdos repercute, sin duda, en ese (por)venir, porque la historia oral, parafraseando a Sebe (2009), más que verse como una colección de acontecimientos apuesta por la transformación de las personas, de la comunidad y de la realidad.

De acuerdo con lo anterior, los recuerdos que podían ser enunciados para dar cuenta de estas identidades podían ser muchos. Allí nos interesaba particularmente uno: el de las memorias de la práctica de la construcción del purradé. Esto, porque teníamos la convicción que un camino para pensar el (por)venir de la educación (matemática) indígena de la comunidad podía rastrearse desde esa práctica.

Nuestro estudio, entonces, tenía que ver con aquello denominado historia oral temática, para lo cual era necesaria la formulación de preguntas bien diseñadas que generaran confianza y complicidad en las conversaciones sostenidas entre los miembros de la comunidad y las investigadoras. Las preguntas tenían que ser pensadas para motivar en el otro el acto mismo de recordar.

Sin embargo, una historia oral temática por sí sola no era suficiente, pues cada uno de los participantes se remitía casi siempre, y sin darse cuenta, a su historia de vida. De esta manera, una combinación entre dos formas de historial oral, esto es, la historia oral temática y la historia oral de vida se tornaron elementos clave en la investigación. En este sentido, Sebe (2000) reconoce: 
Hay proyectos temáticos que combinan algo de historia oral y de historia de vida. En esos casos, lo que se busca es el encuadramiento de los datos objetivos del declarante con las informaciones recogidas. Esa forma de historia oral ha sido muy apreciada porque, al mezclar situaciones vivenciadas, la información gana más vivacidad y sugiere características del narrador. (p. 70).

Esta combinación de formas de hacer historia oral requería, además, valorar la tradición oral como manifestación propia de los pueblos indígenas.

Lo anterior nos posibilitó producir de manera colectiva los siguientes registros: el diario de investigación; las fichas temáticas ${ }^{10}$; los encuentros con el Cabildo; los Círculos de Jaibanás; las entre-vistas; los encuentros con las jóvenes, y las discusiones con los maestros. Registros que nos posibilitaron comprender, de otra forma, no solo el acto mismo de la investigación, sino las formas en que la sabiduría de la comunidad debía ser interpretada y presentada.

\section{Resultados y análisis}

\section{Un análisis deconstructivo de los registros producidos}

Los registros tuvieron necesariamente que ser (re)leídos, (re)estudiados y (re)analizados, considerando los escenarios en que fueron producidos. La comunidad nombraría este proceso como un volver a recorrer ese camino andado, ese camino tejido. Un camino que implicó escuchar muchas veces los audios y los videos de manera colectiva o individual. Ese volver a recorrer nos implicó la transcripción de algunos apartados de los registros producidos. Ese proceso significó, como dice Kvale (2011) "transformar, cambiar de una forma a otra." (p. 124). Transcripciones que resultaron necesarias para comprender los planteamientos implícitos o explícitos de los participantes y, sobre todo, para materializar las conversaciones tejidas pensando en las generaciones (por)venir. En este sentido, dice Sebe (2009):

El trabajo que la Historia Oral puede hacer es, exactamente, este trabajo de auxiliar, de mediar formas de elaboración de estas historias. Pero la historia, el largo viaje de lo oral para lo escrito es un viaje bastante turbulento en términos de posicionamientos. El primero de ellos fue Platón que dice que con la escritura nosotros tenemos la muerte de la memoria, o sea, desde que nosotros registramos por escrito, nosotros no necesitamos más grabar en la memoria y de ahí ese larguísimo proceso de desmemorización que nosotros hacemos. (Encontros SESC memorias- Memória, História Oral e Diferenças, Sebe, 30 de julio de 2009).

10 La tematización es un acto de pensamiento y una técnica de investigación que posibilita abordar los documentos -comprendidos en el sentido de Zuluaga (1999) - para realizar la identificación, la descripción y el análisis de temáticas que se constituyen centrales en una investigación. El instrumento de esta técnica es la ficha temática. Desde las fichas temáticas nos fue posible organizar, sistematizar, establecer relaciones e identificar asuntos planteados por autores y con los cuales fuimos tejiendo diálogos académicos a lo largo de la investigación. 
Registros que, tal vez y como lo afirmó Gilberto Antonio Tascón, posibiliten que la comunidad sea conocida en el futuro por la humanidad:

Hoy nosotros, los indígenas, desde la oralidad, estamos aprendiendo a escribir, para que ese conocimiento quede para la consulta, para el conocimiento de los seres humanos en este planeta Tierra. Creo que eso es lo que estamos haciendo con toda esta construcción que estamos haciendo como estudiantes. (Entrevista de grupos de discusión, Gilberto Antonio Tascón, 20 de abril de 2013).

Bajo estas perspectivas, los registros transcritos posibilitaron, además, poner en diálogo voces nunca antes escuchadas, voces de quienes estaban vivos y de quienes ya habían muerto (voces que pervivían en el tiempo).

Dichos registros transcritos dieron origen a los datos de la investigación desde un análisis deconstructivo. Un análisis que implicó, de acuerdo con Kvale (2011), una combinación de deconstrucción y construcción. La primera, entendida como poner en cuestión, discutir y debatir algunas de las ideas planteadas, y la segunda, asumiendo que tanto las ideas valoradas como aquellas cuestionadas posibilitaron una forma de comprender y de escribir diferente; posibilitaron una reescritura de lo dicho. En palabras de Kvale (2011):

Una lectura deconstructiva desgarra un texto, desestabilizando los conceptos que da por supuestos; se concentra en las tensiones y rupturas de un texto, en lo que un texto pretende decir, así como lo que no se dice en el texto, en lo que el uso de los conceptos del texto excluye. (p. 148).

Sería un error considerar la deconstrucción como el acabamiento de las ideas y de los sujetos que las expresaron. La deconstrucción apunta a una reflexión de lo dicho en el contexto posibilitando, así, la transformación del sujeto que lo expresó, y también la transformación del sujeto que lo puso en duda.

Fueron estos registros producidos, y su análisis deconstructivo, los que posibilitaron la emergencia de tres categorías en el interior de la investigación: "El purradé: İun lugar más que para dormir!"; "La movilización de objetos culturales desde las memorias de la práctica de la construcción del purradé: elementos para la discusión"; "Notas para una problematización transgresiva de la educación (matemática) indígena embera chamí" (Higuita, 2014).

\section{A modo de cierre}

Nuestra intención en este artículo fue visibilizar las tensiones y los desafíos de hacer (y ser) investigación en el campo de la etnomatemática. En este sentido, tornamos visibles tres tensiones existentes en la investigación desde nuestra experiencia. La primera de ellas tuvo que ver con la relación que pudo tejerse entre las investigadoras y la comunidad indígena. La segunda tensión estuvo referida a la necesidad de pensar y considerar la lengua y los diferentes lenguajes producidos por las comunidades para ampliar los horizontes teóricos, metodológicos y prácticos de la investigación en etnomatemática. La tercera y última tensión consistió en la apertura que como investigadores podemos tener para aprender formas otras de ser y estar en el mundo, aprendidas desde la sabiduría de las comunidades indígenas ancestrales.

Los desafíos en la investigación, en este campo, los planteamos a partir del diálogo tejido entre formas propias de hacer investigación desde las comunidades indígenas y los métodos producidos por la cultura occidental, en este caso, desde la historia oral. Así, encontramos en esta última un método que apuesta por la valoración y la comprensión de la experiencia, desde las voces de los que, tradicionalmente, 
han sido silenciados $u$ oprimidos ${ }^{11}$. En este sentido, en esta investigación logramos que los jaibanás, que históricamente habían estado dedicados a proteger el territorio y la salud de los miembros de la comunidad, fueran también convocados para pensar aspectos educativos y para proponer caminos que apuntaran posibilidades para que parte de la sabiduría embera chamí fuese explicitada, discutida y problematizada en la escuela.

La historia oral nos posibilitó tejer diálogos, desde las entre-vistas, con diferentes personas dentro y fuera de la comunidad indígena embera chamí para recorrer y volver a recorrer, conjuntamente, saberes ancestrales de la comunidad que pueden ingresar a la sala de clase de la escuela indígena.

\section{Referencias}

Berrío, K. (2009). La medida en un contexto de escuela indígena: el caso del Pueblo Tule y el caso del pueblo Emberá-Cham [Trabajo de Grado]. Medellín: Licenciatura en Educación Básica con Énfasis en Matemáticas. Universidad de Antioquia.

Bosi, E. (1994). Memória-sonho e memória-trabalho. En E. Bosi, Memória e sociedade (pp. 43-70). São Paulo: Companhia das Letras.

Bosi, E. (2003). A substância social da memória. En E. Bosi. O tempo vivo da memória: Ensaios de piscologia social (pp. 13-48). São Paulo: Ateliê Editorial.

Chilisa, B. (2012). Indigenous research methodologies. Los Ángeles, Londres, Nueva Delhi, Sigapore, Washington: Sage.

Colombia. (1991). Constitución Política de Colombia. Bogotá.

D’Ambrosio, U. (1999). O programa de etnmatemática e questões historiográficas e metodológicas. IV Congreso Brasileiro da Filosofía. São Paulo, Brasil. Recuperado de http://vello.sites.vol.com.br/filosofia.htm.

D’Ambrosio, U. (2008) Etnomatemática. Eslabón entre las tradiciones y la modernidad. México: Limusa.

D'Ambrosio, U. (2011). A busca da paz como responsabilidade dos matemáticos. Cuadernos de investigación y formación en educación matemática, (7). Recuperado de http://www.cimm.ucr.ac.cr/ojs/index.php/CIFEM/article/view/664/653

Freire, P. (2000). Pedagogía del oprimido. México: Siglo XXI Editores.

Freire, P. (2004). Pedagogía de la autonomía. São Paulo: Paz e Terra S.A.

Higuita, C. (201 1). La medida desde la medicina tradicional: el caso de una comunidad Embera Chamí. [Trabajo de Grado]. Medellín: Licenciatura en Educación Básica con Énfasis en Matemáticas. Universidad de Antioquia.

11 Oprimido, en el sentido sugerido por Freire (2000). 
Higuita, C. (2014). La movilización de objetos culturales desde las memorias de la práctica de construcción de la vivienda tradicional embera chamí: posibilidades para pensar el (por)venir de la educación (matemática) indígena. [Disertación de Maestría]. Medellín: Maestría en Educación. Universidad de Antioquia.

Higuita, C. y Jaramillo, D. (2014). La movilización de objetos culturales desde las memorias de la práctica de la construcción del purradé: elementos para otra discusión en educación (matemática) indígena. Revista Latinoamericana de Etnomatemática-RLE: Perspectivas socioculturales de la educación matemática, 7(3), 8-32.

Joutard, P. (1999). Esas voces que nos llegan del pasado. México: Fondo de Cultura Económica.

Kvale, S. (201 1). Las entrevistas en investigación cualitativa. Madrid: Ediciones Morata.

Larrosa, J. (1998). La experiencia de la lectura: estudios sobre literatura y formación. Barcelona: Laertes.

Larrosa, J. (2009). Experiencia y alteridad en Educación. En J. Larrosa y C. Skliar. (comp.), Experiencia y alteridad en educación (pp. 13-44). Rosario: Homo Sapiens Ediciones.

Miguel, A. (2008). Jogos hedonistas de linguagem. São Paulo: Editora Plêiade.

Miguel, A. (2010). Percursos Indisciplinares na Atividade de Pesquisa em História (da Educação Matemática): entre jogos discursivos como práticas e práticas como jogos discursivos. Bolema, 23(35A), 1-57.

Real Academia Española (RAE). (2013). Diccionario de la lengua española. Recuperado de http://rae.es/recursos/diccionarios/drae

Sebe, J. C. (2000). Manual de história oral. São Paulo: Edição Loyola.

Sebe, J. C. (julio de 2009). Memória, história oral e diferenças. Ponencia presentada en Encontros SESC memorias. Recuperado de http://www.youtube.com/watch?$v=Q v P y J-O j s u M$

Smith, L.T. (2012). Caminando sobre terreno resbaladizo: la investigación de los pueblos nativos en la era de la incertidumbre. En N.K. Denzin y Y. Lincoln (coord.). Manual de investigación cualitativa (pp. 190-230). Barcelona: Gedisa.

Thompson, J.B. (201 1). Ideología y cultura moderna: teoria social crítica na era dos meios de comunicação de massa. Petrópolis, RJ: Vozes.

Walsh, C. (2005). Interculturalidad, conocimientos y decolonialidad. Signo y pensamiento 46(24), 39-50.

Walsh, C. (2007a). ¿̇Son posibles unas ciencias sociales/culturales otras? Reflexiones en torno a las epistemologías decoloniales. Nómadas, 26, 102-113. 
Walsh, C. (2007b). Interculturalidad, colonialidad y educación. Educación y Pedagogía, XIX(48), 25-35.

Zuluaga, O. (1999). Pedagogía e historia: la historicidad de pedagogía, la enseñanza, Un objeto de saber. Bogotá: Siglo del Hombre Editores.

\section{Para citar este artículo}

Higuita, C.; Tascón, A. y Jaramillo, D. (2017). La historia oral en las memorias de prácticas culturales para pensar un (por)venir de la educación (matemática) indígena. Tecné, Episteme y Didaxis, TED, 42, 79-94. 